\title{
Nurses' knowledge of stroke-related oropharyngeal dysphagia in the Eastern Cape, South Africa
}

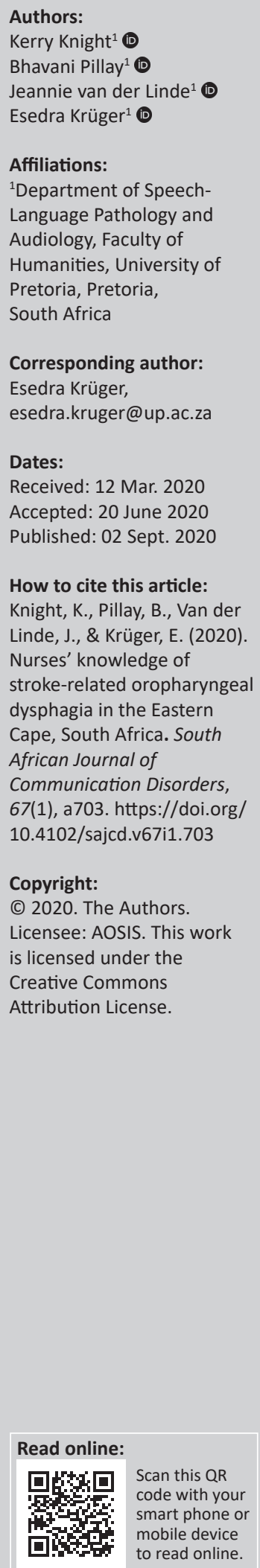

Background: Early identification of stroke-related oropharyngeal dysphagia (OPD) using screening by nurses can prevent adverse patient outcomes in lower middle-income countries. Nurses are essential in the OPD management team and should ideally be able to screen and prioritise dysphagia management in stroke patients.

Objective: The aim of this research was to describe nurses' practices related to identification and management of patients with stroke-related OPD.

Methods: Qualified nurses from various healthcare levels in the Eastern Cape, South Africa were invited to complete a previously published hard copy survey on the signs and symptoms, complications and management of stroke-related OPD. A sample of 130 participants completed the survey.

Results: The mean scores of correct responses for each section were: 8.7/13 (66.7\%) for signs and symptoms, 4.7/10 (47.3\%) for complications and 3.8/7 (54.2\%) for management practices. Statistically, there were no differences between the levels of healthcare for the signs and symptoms section and the complications section. Regarding management of OPD, secondarylevel (S) nurses demonstrated significantly better knowledge than primary-level $(\mathrm{P})$ and tertiary-level $(\mathrm{T})$ nurses $(\mathrm{S}-\mathrm{P}: \mathrm{p}=0.022 ; \mathrm{S}-\mathrm{T}: \mathrm{p}=0.010)$. Secondary-level nurses also scored significantly higher across all three sections (S-P: $p=0.044$; S-T: $p=0.025$ ) than those at the other levels.

Conclusions: The study found that nurses across all levels of healthcare had only moderate knowledge regarding identification and management of stroke-related OPD. Interdisciplinary collaboration between nurses and speech-language therapists may improve nurses' knowledge in identification and management of stroke-related OPD in lower middle-income settings such as South Africa.

Keywords: oropharyngeal dysphagia; dysphagia screening; stroke-related dysphagia; nurse; interdisciplinary collaboration; South Africa; lower middle-income country; survey.

\section{Introduction}

In rural South Africa, strokes result in approximately 30000 deaths yearly and are the largest cause of disability in the country (Maredza, Bertram, Gómez-Olivé, \& Tollman, 2016). Stroke is one of the leading causes of oropharyngeal dysphagia (OPD) (Behera et al., 2018; Joundi et al., 2017). Oropharyngeal dysphagia is often underdiagnosed in people with stroke, leading to adverse complications including aspiration pneumonia, malnutrition, dehydration, extended hospital stay and death (Takizawa, Gemmell, Kenworthy, \& Speyer, 2016).

Early identification of OPD using screening allows for prompt initiation of management plans, which may improve patient outcomes (Sherman et al., 2018).

Nursing staff are more likely to be the first health professionals to detect the early symptoms of OPD as they administer medication and feed patients (Bhimte \& Rangasayee, 2015). People identified with swallowing difficulties should ideally be referred to a speech-language therapist (SLT) for comprehensive assessment and management (American Speech-Language-Hearing Association [ASHA], 2016). Only a limited number of SLTs are employed in the South African public sector, resulting in large caseloads (Ostrofsky \& Seedat, 2016). Contextual challenges of limited resources, both human and physical, are a barrier and often result in long waiting times for the patient and possibly insufficient clinical management of dysphagia cases (Andrews \& Pillay, 2017). The importance of accurate early identification of stroke-related OPD by nurses is thus evident, as this may guide its initial management, such as placing a patient nil per os until 
formal SLT review (Behera et al., 2018; Cummings et al., 2015; Palli et al., 2017). Oropharyngeal dysphagia management may include changes in consistencies, positioning or swallow manoeuvres (South African SpeechLanguage and Hearing Association [SASLHA], 2011).

Ideally, nurses should effectively identify OPD and risk for aspiration by screening patients' swallowing abilities before administering food or liquids (Behera et al., 2018; Palli et al., 2017).

Nurses can implement initial swallowing precautions to prevent unfavourable outcomes until SLTs are able to perform comprehensive swallow assessments (Sherman et al., 2018). Patients who fail a screen and have possible OPD should be referred to an SLT for further assessment (Sherman et al., 2018). In lower-middle-income countries (LMICs) such as South Africa, swallow screenings by nurses are inconsistently conducted because of insufficient screening protocols and limited material and human resources (Blackwell \& Littlejohns, 2010). The result is a lack of clarity as to who has the responsibility to refer to SLTs and which patients require swallowing assessment following stroke (Blackwell \& Littlejohns, 2010).

In South Africa, healthcare professionals may not have a large amount of experience or training in stroke-related OPD (Willie, 2011). A recent unpublished master's study stated that there is also a shortage of nurses in the public sector in South Africa, reducing the amount of time nurses may spend with patients (George, Gow, \& Bachoo, 2013; Robbertse, 2018). Only 50\% of nurses service the majority of patients in an under-resourced setting (Blackwell \& Littlejohns, 2010). Nurses who receive sufficient training and support to perform dysphagia screenings are more likely to use swallow screening protocols and appropriately refer to SLTs (Sivertsen, Graverholt, \& Espehaug, 2017). Yet international studies report that nurses display difficulty identifying OPD timeously following a stroke, delaying appropriate referral for intervention (Bhimte \& Rangasayee, 2015).

Nursing staff in South Africa demonstrated low awareness of the role of SLTs in OPD management, possibly because of insufficient undergraduate training on OPD and minimal interaction with SLTs in practice (Albini, Soares, Wolf, \& Gonçalves, 2013; Robbertse, 2018).

Prevention of dysphagia-related complications and establishment of screening protocols to clearly indicate the roles and responsibilities of various professionals in a dysphagia team is the role of SLTs (ASHA, 2016). The SLT's involvement in interdisciplinary teamwork and collaboration with nurses could ensure that team members are informed and adequately prepared for identification of OPD (Rhoda \& Pickel-Voight, 2015). More regular interaction between nurses and SLTs can allow for sharing of knowledge and expertise between professions (Robbertse,
2018), which is essential within LMICs (Blackwell \& Littlejohns, 2010). Improved interdisciplinary collaboration may lead to shorter hospital stays, lessen the number of patient complications and decrease mortality rates in people with stroke-related OPD (Dondorf, Fabus, \& Ghassemi, 2015). The aim of this study was to describe nurses' knowledge of stroke-related OPD in the Eastern Cape, South Africa which may identify possible training needs and guide future interprofessional collaboration between SLTs and nurses in this setting.

\section{Methods \\ Study design and setting}

Data were collected using a validated survey on eating and swallowing difficulties(Rhoda \& Pickel-Voight, 2015) and a quantitative descriptive study design (Leedy \& Ormrod, 2014). The survey was distributed by hand to 135 nurses from five sites in a metropolitan area of the Eastern Cape, South Africa. The study aimed to gather data in three areas: the identification, complications and management of strokerelated OPD.

\section{Study population and sampling}

Participants were required to be qualified nurses working with people with stroke, who were proficient in English. Using purposive sampling, 135 participants were recruited (Leedy \& Ormrod, 2014). Three surveys were returned incomplete, and two surveys were not returned; therefore, the responses of 130 participants were analysed. Participant characteristics are presented in Table 1. Approximately half

TABLE 1: Participants' socio-demographic characteristics and professional experience $(N=130)$

\begin{tabular}{|c|c|c|}
\hline Characteristic & $n$ & $\%$ \\
\hline \multicolumn{3}{|l|}{ Level of healthcare } \\
\hline Primary & 44 & 33.8 \\
\hline Secondary & 50 & 38.5 \\
\hline Tertiary & 36 & 27.7 \\
\hline \multicolumn{3}{|l|}{ Age (years) } \\
\hline 20-30 & 21 & 16.2 \\
\hline $31-40$ & 28 & 21.5 \\
\hline $41-50$ & 39 & 30.0 \\
\hline $51-60$ & 41 & 31.5 \\
\hline Over 60 & 1 & 0.8 \\
\hline \multicolumn{3}{|l|}{ Qualification } \\
\hline Enrolled nursing assistant & 29 & 22.3 \\
\hline Staff nurse & 21 & 16.2 \\
\hline Professional nurse & 80 & 61.5 \\
\hline \multicolumn{3}{|l|}{ Years of nursing experience } \\
\hline $0-3$ & 17 & 13.0 \\
\hline $4-6$ & 27 & 20.8 \\
\hline 7-9 & 20 & 15.4 \\
\hline $10+$ & 66 & 50.8 \\
\hline \multicolumn{3}{|l|}{ Current work context $\dagger$} \\
\hline Medical ward & 86 & 66.2 \\
\hline Casualty & 7 & 5.4 \\
\hline Out-patient clinic & 20 & 15.4 \\
\hline Day hospital & 24 & 18.5 \\
\hline
\end{tabular}

$\dagger$, Participants may be actively working in more than one context. 
the participants $(n=66 ; 50.8 \%$ ) had 10 or more years of experience, and 86 participants $(66.2 \%)$ were working in medical wards (Table 1).

\section{Data collection}

A published survey about eating and swallowing difficulties (Rhoda \& Pickel-Voight, 2015) was used to collect data. The survey was previously validated by five practising SLTs with experience in assessment and management of OPD, and the test-retest method was used to ensure reliability (Rhoda \& Pickel-Voight, 2015). The survey consisted of four sections: demographic information and level of experience, signs and symptoms, complications and management of dysphagia. A pilot study was conducted with 10 participants prior to data collection to ensure clarity of questions and increasing reliability of the findings. Data were collected from July to September 2018 by the first author, who distributed the hard copy surveys by hand and was available for clarification. Nurses who did not wish to participate were able to refuse participation from the outset or withhold submission of the survey.

\section{Data analysis}

The survey was scored using the guidelines of Rhoda and Pickel-Voight (2015), tallying the number of correct responses in each section. Each participant's level of knowledge was classified as follows: A score of $\geq 75 \%$ was 'high', 50\% - 74\% was 'moderate' and a score of $\leq 50 \%$ was 'low' (Rhoda \& Pickel-Voight, 2015). Data were analysed using the Statistical Package for the Social Sciences version 25 , using descriptive and inferential statistics in consultation with a statistician. The Cronbach's alpha coefficient equalled 0.707, demonstrating the reliability of the questionnaire (Goforth, 2015). P-values of $<0.05$ were considered statistically significant. Variability of responses was noted across nurses and within levels.

\section{Ethical considerations}

Ethical clearance was obtained from Faculty of Humanities and Health Sciences, University of Pretoria (protocol number GW20180105HS). Permission was obtained from the required provincial Department of Health governing bodies, with voluntary participation and written informed consent from each participant.

\section{Results}

\section{Level of experience}

Most participants $(n=118 ; 90.8 \%)$ had cared for a patient who had had a stroke, whereas only $50 \%(n=65)$ had received training on stroke patient care. The majority of participants ( $n=100 ; 76.9 \%$ ) had cared for stroke patients with swallowing difficulties, with only $26.9 \%(n=35)$ having received training on OPD and its management. Interestingly, $81.5 \%(n=106)$ of participants indicated that they were unsatisfied with their current knowledge of stroke-related OPD, and most participants $(n=125 ; 96.2 \%)$ indicated a desire for further training. Variability of responses was noted across participants and within the different levels of healthcare.

\section{Identification of signs and symptoms of oropharyngeal dysphagia}

Table 2 shows a comparison of participants' understanding of the typical symptoms of OPD. In Tables 2 to 4 , the $p$-values labelled ' $\mathrm{P}-\mathrm{S}$ ' (primary- and secondary-level groups), ' $\mathrm{P}-\mathrm{T}$ ' (primary- and tertiary-level groups), and ' $\mathrm{S}-\mathrm{T}$ ' (secondaryand tertiary-level groups) indicate whether correct responses differed significantly between groups.

As a group, participants performed best in the identification section, with a mean correct response score of $8.7 / 13$, indicating that $66.7 \%$ of the symptoms were identified correctly. However, when asked whether patients always cough if they aspirate, only $5.4 \%(n=7)$ of participants

TABLE 2: Comparison of participants' knowledge of signs and symptoms of oropharyngeal dysphagia $(N=130)$.

\begin{tabular}{|c|c|c|c|c|c|c|c|c|c|c|c|c|}
\hline \multirow[t]{2}{*}{ Signs/symptoms } & \multirow{2}{*}{$\begin{array}{l}\text { Correct } \\
\text { answer }\end{array}$} & \multicolumn{2}{|c|}{ Primary $(n=44)$} & \multicolumn{2}{|c|}{ Secondary $(n=50)$} & \multicolumn{2}{|c|}{ Tertiary $(n=36)$} & \multicolumn{3}{|c|}{ Fisher's exact test $(p)$} & \multicolumn{2}{|c|}{ Total } \\
\hline & & $n$ & $\%$ & $n$ & $\%$ & $n$ & $\%$ & P-S & $\mathbf{P}-\mathbf{T}$ & S-T & $n$ & $\%$ \\
\hline Coughing whilst eating & A & 36 & 81.8 & 46 & 92.0 & 32 & 88.9 & 0.215 & 0.532 & 0.715 & 114 & 87.7 \\
\hline Skin irritations & $\mathrm{D}$ & 30 & 68.2 & 33 & 66.0 & 20 & 55.6 & 1.000 & 0.258 & 0.373 & 83 & 63.8 \\
\hline $\begin{array}{l}\text { Feeling of food getting stuck in } \\
\text { throat }\end{array}$ & A & 38 & 86.4 & 45 & 90.0 & 29 & 80.6 & 0.750 & 0.551 & 0.227 & 112 & 86.2 \\
\hline $\begin{array}{l}\text { Choking on saliva during } \\
\text { non-mealtimes }\end{array}$ & A & 37 & 84.1 & 44 & 88.0 & 28 & 77.8 & 0.766 & 0.569 & 0.244 & 109 & 83.8 \\
\hline Poor movement of tongue & A & 41 & 93.2 & 43 & 86.0 & 31 & 86.1 & 0.327 & 0.457 & 1.000 & 115 & 88.5 \\
\hline Food remains in mouth & $A$ & 36 & 81.8 & 47 & 94.0 & 33 & 91.7 & 0.106 & 0.329 & 0.691 & 116 & 89.2 \\
\hline Poor chewing & A & 37 & 84.1 & 44 & 88.0 & 33 & 91.7 & 0.766 & 0.499 & 0.729 & 114 & 87.7 \\
\hline $\begin{array}{l}\text { Patients always cough if they } \\
\text { aspirate }\end{array}$ & $\mathrm{D}$ & 3 & 6.8 & 3 & 6.0 & 1 & 2.8 & 1.000 & 0.623 & 0.637 & 7 & 5.4 \\
\hline Difficulty closing lips & A & 37 & 84.1 & 33 & 66.0 & 14 & 38.9 & 0.059 & $0.000 *$ & $0.016^{*}$ & 84 & 64.6 \\
\hline Weight loss & A & 32 & 72.7 & 33 & 66.0 & 21 & 58.3 & 0.510 & 0.236 & 0.504 & 86 & 66.2 \\
\hline $\begin{array}{l}\text { Frequent throat clearing after } \\
\text { swallowing }\end{array}$ & A & 29 & 65.9 & 31 & 62.0 & 18 & 50.0 & 0.830 & 0.176 & 0.280 & 78 & 60.0 \\
\hline Hoarse voice & A & 25 & 56.8 & 31 & 62.0 & 8 & 22.2 & 0.676 & $0.003 *$ & $0.000 *$ & 64 & 49.2 \\
\hline Chest pain & A & 11 & 25.0 & 18 & 36.0 & 17 & 47.2 & 0.272 & 0.059 & 0.375 & 46 & 35.4 \\
\hline
\end{tabular}

A, agree; D, disagree; P, primary; S, secondary; $T$, tertiary.

Note: Results indicate number (\%) of correct responses.

*, Statistically significant difference between number of correct responses between the two groups. 
responded correctly. Two other symptoms also generated low scores, namely 'hoarse voice' $(49.2 \% ; n=64)$ and 'chest pain' $(35.4 \% ; n=46)$. More common symptoms such as 'coughing whilst eating' ( $n=114 ; 87.7 \%)$, 'poor movement of the tongue' $(n=115 ; 88.5 \%)$ and 'food remaining in the mouth' $(n=116$; $89.2 \%$ ) were accurately identified by most participants.

Statistically, there were no significant differences across nurses working at the various levels of healthcare in this section $(p=0.066)$. However, for the item 'difficulty closing lips', the percentage of correct responses differed significantly, with primary-level nurses scoring a high correct response rate $(84.1 \%)$ and tertiary-level nurses scoring much lower $(38.9 \% ; p=0.000)$. There was also a significant difference for this item between secondary-level nurses, with the next highest score $(66.0 \%)$, and tertiarylevel nurses (38.9\%; $p=0.016)$. Interestingly, primary-level nurses $(56.8 \%)$ had significantly more correct responses to the item 'hoarse voice' when compared to the tertiary-level nurses $(22.2 \% ; p=0.003)$. The same trend was seen for this item amongst secondary-level nurses (62.0\%), who demonstrated significantly more correct responses than the tertiary-level nurses $(22.2 \% ; p=0.000)$.

\section{Complications of oropharyngeal dysphagia}

Participants scored the lowest in this section of the survey, with a mean correct score of $4.73 / 10$, revealing that only $47.3 \%$ of complications were accurately identified (Table 3 ).
Three complications were incorrectly thought to be the result of a stroke, with only a small percentage of participants identifying them correctly (Table 3). These were 'anaphylactic shock' ( $n=32 ; 24.6 \%)$, 'problems with digestion' $(n=11 ; 8.5 \%)$ and 'sudden heart attack' ( $n=36 ; 27.7 \%)$. Professional nurses performed better in this section when compared to enrolled nursing assistants $(p=0.015)$ and to staff nurses $(p=0.020)$. Table 3 shows that participants were not familiar with OPD complications that could arise following stroke. For the item 'increased mortality', primary-level nurses demonstrated the best knowledge $(70.5 \%)$ when compared to tertiary-level nurses $(30.6 \% ; p=0.001)$. However, the tertiary-level nurses (58.0\%) also scored significantly lower on this item compared to the secondary-level nurses $(p=0.016)$. A similar result was found for 'general weakness', where both primary-level $(86.4 \%)$ and secondary-level $(82.0 \%)$ nurses had high scores, demonstrating better knowledge than the tertiary-level nurses (61.1\%). The correct response rate for 'sudden heart attack' was low across groups, with the tertiary-level nurses having the highest score (38.9\%) and primary-level the lowest (15.9\%). For 'haematemesis', the tertiary-level nurses had the best knowledge (55.6\%), and the primary-level nurses had the fewest correct responses $(25.0 \%)$.

\section{Management practices for oropharyngeal dysphagia}

Participants were required to determine whether or not certain management practices were appropriate (Table 4).

TABLE 3: Comparison of participants' knowledge of complications of oropharyngeal dysphagia $(N=130)$.

\begin{tabular}{|c|c|c|c|c|c|c|c|c|c|c|c|c|}
\hline \multirow[t]{2}{*}{ Complications } & \multirow{2}{*}{$\begin{array}{l}\text { Correct } \\
\text { answer }\end{array}$} & \multicolumn{2}{|c|}{ Primary $(n=44)$} & \multicolumn{2}{|c|}{ Secondary $(n=50)$} & \multicolumn{2}{|c|}{ Tertiary $(n=36)$} & \multicolumn{3}{|c|}{ Fisher's exact } & \multicolumn{2}{|c|}{ Total $(n=130)$} \\
\hline & & $n$ & $\%$ & $n$ & $\%$ & $n$ & $\%$ & P-S & $\mathbf{P}-\mathbf{T}$ & S-T & $n$ & $\%$ \\
\hline Increased mortality & $A$ & 31 & 70.5 & 29 & 58 & 11 & 30.6 & 0.282 & $0.001 *$ & $0.016 *$ & 71 & 54.6 \\
\hline Pneumonia & A & 23 & 52.3 & 30 & 60 & 15 & 41.7 & 0.533 & 0.376 & 0.126 & 68 & 52.3 \\
\hline Anaphylactic shock & $\mathrm{D}$ & 9 & 20.5 & 12 & 24 & 11 & 30.6 & 0.805 & 0.314 & 0.622 & 32 & 24.6 \\
\hline General weakness & A & 38 & 86.4 & 41 & 82 & 22 & 61.1 & 0.588 & $0.018^{*}$ & $0.047 *$ & 101 & 77.7 \\
\hline Problems with digestion & $\mathrm{D}$ & 2 & 4.5 & 4 & 8 & 5 & 13.9 & 0.681 & 0.234 & 0.482 & 11 & 8.5 \\
\hline Dehydration & $A$ & 33 & 75 & 44 & 88 & 27 & 75 & 0.116 & 1.000 & 0.153 & 104 & 80 \\
\hline Sudden heart attack & $\mathrm{D}$ & 7 & 15.9 & 15 & 30 & 14 & 38.9 & 0.144 & $0.013 *$ & 0.489 & 36 & 27.7 \\
\hline Malnutrition & $A$ & 36 & 81.8 & 39 & 78 & 30 & 83.3 & 0.798 & 1.000 & 0.594 & 105 & 80.8 \\
\hline Haematemesis (vomiting blood) & $\mathrm{D}$ & 11 & 25 & 21 & 42 & 20 & 55.6 & 0.126 & $0.006 *$ & 0.275 & 52 & 40 \\
\hline
\end{tabular}

Note: Results indicate the number (\%) of correct responses.

*, Statistically significant difference between number of correct responses between the two groups: P = Primary, S = Secondary, T = Tertiary A, Agree; D, Disagree; P, Primary; S, Secondary; T, Tertiary.

TABLE 4: Comparison of participants' knowledge of the management of OPD $(N=130)$.

\begin{tabular}{|c|c|c|c|c|c|c|c|c|c|c|c|c|}
\hline \multirow[t]{2}{*}{ Management practices } & \multirow[t]{2}{*}{$\begin{array}{l}\text { Correct } \\
\text { answer }\end{array}$} & \multicolumn{2}{|c|}{$\begin{array}{l}\text { Primary } \\
(n=44)\end{array}$} & \multicolumn{2}{|c|}{$\begin{array}{l}\text { Secondary } \\
(n=50)\end{array}$} & \multicolumn{2}{|c|}{$\begin{array}{l}\text { Tertiary } \\
(n=36)\end{array}$} & \multicolumn{3}{|c|}{$\begin{array}{l}\text { Fisher's exact } \\
p \text {-value }\end{array}$} & \multicolumn{2}{|c|}{ Total $(n=130)$} \\
\hline & & $n$ & $\%$ & $n$ & $\%$ & $n$ & $\%$ & P-S & P-T & S-T & $n$ & $\%$ \\
\hline $\begin{array}{l}\text { Patients with a nasogastric tube need daily oral } \\
\text { hygiene (mouth washing and brushing of the teeth) }\end{array}$ & A & 42 & 95.5 & 48 & 96 & 33 & 91.7 & 1.000 & 0.653 & 0.645 & 123 & 94.6 \\
\hline Thickened liquid should be avoided & D & 6 & 13.6 & 21 & 42 & 5 & 13.9 & $0.003 *$ & 1.000 & $0.008^{*}$ & 32 & 24.6 \\
\hline Watery liquids are the safest to drink & $\mathrm{D}$ & 2 & 4.5 & 17 & 34 & 11 & 30.6 & $0.001^{*}$ & $0.002 *$ & 0.818 & 30 & 23.1 \\
\hline $\begin{array}{l}\text { All patients with difficulty swallowing need a } \\
\text { feeding tube }\end{array}$ & $\mathrm{D}$ & 19 & 43.2 & 17 & 34 & 5 & 13.9 & 0.4 & $0.007^{*}$ & $0.046^{*}$ & 41 & 31.5 \\
\hline $\begin{array}{l}\text { The best position while feeding the patient is } \\
\text { when the patient lies flat on his back }\end{array}$ & $\mathrm{D}$ & 37 & 84.1 & 46 & 92 & 31 & 86.1 & 0.337 & 1.000 & 0.482 & 114 & 87.7 \\
\hline The patient can always eat normal hospital food & $\mathrm{D}$ & 37 & 84.1 & 40 & 80 & 22 & 61.1 & 0.789 & $0.024 *$ & 0.087 & 99 & 76.2 \\
\hline $\begin{array}{l}\text { A feeding tube is only indicated in a patient with } \\
\text { impaired consciousness }\end{array}$ & D & 16 & 36.4 & 23 & 46 & 15 & 41.7 & 0.404 & 0.652 & 0.826 & 54 & 41.5 \\
\hline
\end{tabular}

Note: Results indicate the number (\%) of correct responses.

*, Statistically significant difference between number of correct responses between the two groups: A, Agree; D, Disagree; P, Primary; S, Secondary; T, Tertiary. 
Participants had a mean correct score of $3.8 / 7$, reflecting an average of $54.2 \%$ for this section, indicating some difficulty identifying ideal management practices for patients with stroke-related OPD.

A few misconceptions were evident, indicated by low percentages of correct responses of disagreement with inappropriate management practices. These included that 'thickened liquids should be avoided' ( $n=32 ; 24.6 \%)$, 'watery liquids are the safest to drink' $(n=30 ; 23.1 \%)$ and 'all patients with difficulty swallowing require a feeding tube' ( $n=41 ; 31.5 \%)$. Remarkably, the secondary-level nurses had better knowledge on the item 'thickened liquid should be avoided' (42.0\%) when compared to both primarylevel (13.6\%) and tertiary-level (13.9\%) nurses. For 'watery liquids are the safest to drink', the secondary-level (34.0\%) and tertiary-level (30.6\%) nurses scored similarly, whereas the primary-level nurses had a low score $(4.5 \%)$. On the item 'all patients with difficulty swallowing need a feeding tube', the primary-level nurses scored the highest (43.2\%), followed by the secondary-level nurses $(34.0 \%)$, and the tertiary-level nurses scored the lowest (13.9\%).

Finally, a significant difference was found for 'the patient can always eat normal hospital food' between the primary $(84.1 \%)$ and tertiary $(61.1 \%)$ levels $(p=0.024)$.

Statistically, for the management of OPD section, the secondary-level nurses showed significantly better knowledge when compared to the primary-level nurses $(p=0.022)$ and the tertiary-level nurses $(p=0.010)$. The same is seen in the overall number of correct responses, with the secondary-level nurses displaying the best knowledge on OPD, when compared to the primary-level nurses (S-P: $p=0.044)$ and the tertiary level nurses (S-T: $p=0.025)$. The study revealed moderate scores overall across all levels of healthcare with a mean correct score of $17.20 / 30$ (57.33\%).

\section{Discussion}

Despite regularly managing stroke patients, this study revealed that only half of the participants had received previous training on stroke, and only $26.9 \%$ of the participants had received training on stroke-related OPD.

Although research shows that OPD occurs in up to $80 \%$ of persons who have suffered a stroke (Takizawa et al., 2016), the nurses in this study, who are vital in the management of OPD, had received minimal training.

In order to manage OPD, it is important for nurses to have good knowledge of OPD symptoms for early identification and avoidance of unfavourable outcomes such as malnutrition, aspiration pneumonia and death (Behera et al., 2018; Palli et al., 2017). The results revealed that all three levels of nurses had better knowledge of the symptoms than about the complications and management of OPD. Interestingly, tertiary-level participants had the lowest scores for most items in this section, which may be attributed to the management of stroke patients in the South African healthcare system: Patients first present with a stroke at local clinics and are referred up the levels of healthcare as necessary; once medically stable, patients are often transferred back to a lower healthcare level for chronic management (KwaZulu-Natal Department of Health, 2014). Therefore, tertiary- and secondary-level nurses often only manage acute stroke patients with OPD.

Despite good knowledge of symptoms as a group, there was a misconception amongst participants on coughing as a sign of aspiration. Patients do not always cough when they aspirate (Miles et al., 2013); however, 83.1\% $(n=108)$ of participants believed coughing was always present, indicating poor awareness of silent aspiration, a well-known symptom of stroke-related OPD (Felix, Joseph, \& Daniels, 2019). Similar results were found by Rhoda and Pickel-Voight (2015), with $80.4 \%$ of nurses believing that coughing was always present during aspiration. If unidentified, silent aspiration places patients at risk for aspiration pneumonia (Teuschl et al., 2018). Therefore, it is essential for nurses across all levels of healthcare to be aware of all symptoms of OPD to effectively manage patients.

Only $52.3 \%$ of participants believed that pneumonia was a complication of OPD. A recent study indicated that stroke patients diagnosed with OPD are 4.69 times more likely to get aspiration pneumonia than those without OPD (Feng et al., 2019). For this reason, it is vital that nurses be aware of complications associated with mismanaged OPD and have knowledge of the importance of appropriate management. The participants had a mean correct score of $4.73 / 10$ for identifying the complications associated with stroke-related OPD, indicating that nurses across all levels of healthcare in this study were unaware of adverse consequences resulting from undiagnosed or mismanaged OPD. The responses varied widely between items as well as between healthcare levels, which may result from the varying caseloads that nurses face. Similarly, primary-level nurses were the most accurate in identifying both increased mortality $(70.5 \%)$ and general weakness $(86.4 \%)$, followed by secondary-level $(58.0 \%$ and $82.0 \%$, respectively) and tertiary-level nurses with the lowest scores $(30.6 \%$ and $61.1 \%$, respectively) for these complications.

Most participants $(n=89 ; 68.5 \%)$ believed that all patients with OPD should be fed using nasogastric tubes, which may be because it is viewed as the predominant management option for stroke-related OPD. Secondary-level nurses had the best knowledge of the correct management procedures for patients with stroke-related OPD.

Overall, secondary-level nurses had the highest scores $(\mathrm{P}=17.80 / 30 ; \mathrm{S}=18.94 / 30 ; \mathrm{T}=16.50 / 30)$ across all sections of the survey and therefore had the best knowledge of stroke-related OPD.

Nursing staff will most often be feeding or providing medication to persons with stroke; thus, knowledge of the 
delivery method of food and liquids, food consistency and positioning of patient is crucial (Bhimte \& Rangasayee, 2015). Most participants (75.4\%) were unaware of the value of thickening agents in the treatment of OPD. Thickening agents do not improve swallow physiology, but research emphasises the use of thickeners as a compensatory measure to improve swallow safety and reduce aspiration risk by slowing the flow of the bolus (Cichero et al., 2017).

A South African study conducted in two provinces found that nurses identified limited staff and time, as well as insufficient knowledge and training on OPD, as barriers to appropriate OPD management (Robbertse, 2018). The same can be seen in the current study, where the inconsistencies and misconceptions about OPD demonstrate a need for more knowledge and training. Similar results regarding knowledge and training across all sections of the survey were found by Rhoda and Pickel-Voight (2015). Nurses in the current study, as well as the study by Rhoda and Pickel-Voight (2015), demonstrated the best knowledge in the signs and symptoms of stroke-related OPD section of the survey. The previous study found a score of $64.62 \%$, compared to $66.7 \%$ in this study. In contrast to the previous study, where the next best outcome was of the complications associated with stroke-related OPD $(58.15 \%)$, nurses in this research had the fewest correct responses $(47.3 \%)$.

Lastly, nurses in the previous study scored $49.28 \%$ for management of stroke-related OPD, in comparison to $54.2 \%$ in the current study.

According to the classification of scores, both studies revealed that nurses had moderate knowledge of stroke-related OPD, with previous research reporting a score of $57.34 \%$ and this study reporting $57.33 \%$. This finding may indicate a need for improved training and opportunities for continued collaboration between SLTs and nurses for improving management of stroke-related OPD so as to optimise patient outcomes. It is essential to develop effective training opportunities on OPD in multiple modes and facilitate regular interaction between nurses and SLTs to improve the quality of patient care (Robbertse, 2018). Collaboration between SLTs and nurses, combining nurses' experience with SLTs' knowledge and expertise on stroke-related OPD, can be used to maximise health resources in a constrained environment in order to improve the interdisciplinary management of stroke patients with OPD in LMICs (Dondorf et al., 2015).

\section{Limitations and future recommendations}

The research was conducted in only one municipality in the Eastern Cape, South Africa comprising three levels of healthcare. As there is limited research available in the local context regarding nurses' knowledge and needs relating to OPD, reference was made to, and comparisons drawn from, an unpublished master's study, given its relevance to this study. The data were collected in a metropolitan area, further limiting the generalisability of the findings. Larger-scale studies of a similar nature across several provinces are recommended.
Further investigation may be done on the availability of training programs for nursing professionals, and support offered in hospitals regarding decisions for patients with stroke-related OPD. Research should aim to identify existing resources to implement better stroke-related OPD identification and management procedures in hospitals.

\section{Conclusion}

The study revealed that secondary-level nurses had better knowledge of symptoms and management of stroke-related OPD than primary- or tertiary-level nurses. The findings highlighted nurses' misconceptions about the possible complications associated with stroke-related OPD. Speechlanguage therapists need to assist in the development and implementation of sustainable protocols for OPD screening and management within resource-constrained environments such as the South African health sector.

Integrated work relations with an emphasis on collaboration between nurses and SLTs will optimise the use of limited health resources, promote patient safety and improve outcomes (Dondorf et al., 2015).

\section{Acknowledgements}

The authors thank Prof. Marien Graham (statistical analysis) and Dr Stacey Rossouw (support of the project).

\section{Competing interests}

The authors have declared that no competing interests exist.

\section{Authors' contributions}

K.K. conceptualised the project, collected and analysed data, and wrote the manuscript. E.K., B.P. and J.V.L. conceptualised the project and wrote the manuscript.

\section{Funding information}

Kerry Knight received a University of Pretoria scholarship.

\section{Data availability statement}

Data are stored at the University of Pretoria for a period of 15 years as per university regulations.

\section{Disclaimer}

The views and opinions expressed in this article are those of the authors and do not necessarily reflect the official policy of any affiliated agency of the authors.

\section{References}

Albini, R.M.N., Soares, V.M.N., Wolf, A.E., \& Gonçalves, C.G. (2013). Knowledge of nursing professionals about the care to dysphagic patients in intensive care units. Revista CEFAC, 15(6), 1512-1524. https://doi.org/10.1590/S151618462013005000047

American Speech-Language-Hearing Association (ASHA). (2016). Scope of practice in speech-language pathology. Retrieved from https://www.asha.org/policy/ SP2016-00343/ 
Andrews, M., \& Pillay, M. (2017). Poor consistency in evaluating South African adults with neurogenic dysphagia. SAJCD, 64(1), e1-e14. https://doi.org/10.4102/sajcd. v64i1.158.

Behera, A., Read, D., Jackson, N., Saour, B., Alshekhlee, D., \& Mosier, A.K. (2018). A validated swallow screener for dysphagia and aspiration in patients with stroke. Journal of Stroke and Cerebrovascular Disorders, 27(7), 1-8. https://doi.org/ 10.1016/j.jstrokecerebrovasdis.2018.02.037

Bhimte, S., \& Rangasayee, R. (2015). To study awareness level of dysphagia and role of speech language pathologist in dysphagia assessment and management among nurses. Journal of Medical, Scientific and Clinical Research, 3(7), 6676-6681. https://doi.org/10.4172/2167-7182.1000213

Blackwell, Z., \& Littlejohns, P. (2010). A review of the management of dysphagia: A South African perspective. Journal of Neuroscience and Nurses, 42(2), 61-70. https://doi.org/10.1097/JNN.0b013e3181ce5c86

Cichero, J.A.Y., Lam, P., Steele, C.M., Hanson, B., Chen, J., Dantas, R.O., ... Stanschus, S. (2017). Development of international terminology and definitions for texturemodified foods and thickened fluids used in dysphagia management: The IDDS framework. Dysphagia, 32(2), 293-314. https://doi.org/10.1007/s00455-0169758-y

Cummings, J., Soomans, D., O' Laughlin, J., Snapp, V., Jodoin, A., Proco, H., Archer, M. \& Rood, D. (2015). Sensitivity and Specificity of a Nurse Dysphagia Screen in Stroke Patients. MEDSURG Nursing, 24(4), 219-222.

Dondorf, K., Fabus, R., \& Ghassemi, A.E. (2015). The interprofessional collaboration between nurses and speech-language pathologists working with patient diagnosed with dysphagia in skilled nursing facilities. Journal of Nursing Education and Practice, 6(4), 17-20. https://doi.org/10.5430/jnep.v6n4p17

Felix, C.C., Joseph, M.E., \& Daniels, S.K. (2019). Clinical decision making in patients with stroke-related dysphagia. Seminars in Speech and Language, 40(3), 188-202. https://doi.org/10.1055/s-0039-1688815

Feng, M.C., Lin, Y.C., Chang, Y.H., Chen, C.-H., Chiang, H.-C., Huang, L.-C., ... Hung C.-H. (2019). The mortality and the risk of aspiration pneumonia related with dysphagia in stroke patients. Journal of Stroke and Cerebrovascula Disease, 28(5),1381-1387. https://doi.org/10.1016/j.jstrokecerebrovasdis.2019. 02.011

George, G., Gow, J., \& Bachoo, S. (2013). Understanding the factors influencing healthworker employment decisions in South Africa. Human Resources for Health, 11(15), 1-7. https://doi.org/10.1186/1478-4491-11-15

Goforth, C. (2015). Using and interpreting Cronbach's alpha. University of Virginia library. Retrieved from http://data.library.virginia.edu/using-and-interpretingcronbachs-alpha/

Joundi, R.A., Martino, R., Saposnik, G., Giannakeas, V., Fang, J., \& Kapral, M.K. (2017) Predictors and outcomes of dysphagia screening after acute ischemic stroke. Stroke, 48(4), 900-906. https://doi.org/10.1161/STROKEAHA.116.015332
Kwazulu Natal Department of Health. (2014). Referral system. Corporate communication. Retrieved from http://www.kznhealth.gov.za/Referral-system.htm

Leedy, P.D., \& Ormrod, J.E. (2014). Practical research: Planning and design (10th edn.). Essex: Pearson Education Limited.

Maredza, M., Bertram, M.Y., Gómez-Olivé, X.F., \& Tollman, S.M. (2016). Burden of stroke attributable to selected lifestyle risk factors in rural South Africa. BMC Public Health, 16(1), 143. https://doi.org/10.1186/s12889-016-2805-7

Miles, A., Moore, S., McFarlane, M., Lee, F., Allen, J., \& Huckabee, M.L. (2013). Comparison of cough reflex test against instrumental assessment of aspiration. Physiology and Behaviour, 118, 25-31. https://doi.org/10.1016/j.physbeh.2013.05.004

Ostrofsky, C., \& Seedat, J. (2016). The South African dysphagia screening tool (SADS): A screening tool for a developing context. South African Journal of Communication Disorders, 63(1), 1-9. https://doi.org/10.4102/sajcd.v63i1.117

Palli, C., Fandler, S., Doppelhofer, K., Niederkorn, K., Enzinger, C., Vetta, C., ... Gattringer, T. (2017). Early dysphagia screening by trained nurses reduces pneumonia rate in stroke patients: A clinical intervention study. Stroke, 48(9), 2583-2585. https://doi.org/10.1161/STROKEAHA.117.018157

Rhoda, A., \& Pickel-Voight, A. (2015). Knowledge of nurses regarding dysphagia in patients post stroke in Namibia. Curationis, 38(2), 1564-1571. https://doi:10.4102/curationis.v38i2.1564

Robbertse, A. (2018). Nurses' perceptions of barriers to care for patients with dysphagia and their information delivery preferences regarding dysphagia care. Unpublished Masters dissertation, University of Stellenbosch.

Sherman, V., Flowers, H., Kapral, M.K., Nicholson, G., Silver, F., \& Martino, R. (2018) Screening for dysphagia in adult patients with stroke: Assessing the accuracy of informal detection. Dysphagia, 33(5), 662-669. https://doi.org/10.1007/s00455018-9885-8

Sivertsen, J., Graverholt, B., \& Espehaug, B. (2017). Dysphagia screening after acute stroke: A quality improvement project using criteria-based clinical audit. BMC Nursing, 16(1), 27. https://doi.org/10.1186/s12912-017-0222-6

South African Speech-Language and Hearing Association (SASLHA). (2011). Guidelines: Dysphagia in adults. Johannesburg: South African Speech Language and Hearing Association.

Takizawa, C., Gemmell, E., Kenworthy, J., \& Speyer, R. (2016). A systematic review of the prevalence of oropharyngeal dysphagia in stroke, Parkinson's disease, Alzheimer's disease, head injury, and pneumonia. Dysphagia, 31(3), 434-441. https://doi.org/10.1007/s00455-016-9695-9

Teuschl, Y., Trapl, M., Ratajczak, P., Matz, K., Dachenhausen, A., \& Brainin, M. (2018). Systematic dysphagia screening and dietary modifications to reduce

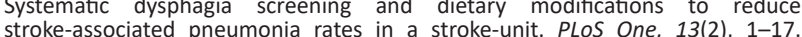
stroke-associated pneumonia rates in a stroke-u
https://doi.org/10.1371/journal.pone.0192142

Willie, M.M. (2011). Access to public health facilities: Taking stock of South Africa's rural Eastern Cape Province. World Medical Journal, 57(5), 164-170. 